\title{
An attempt to derive the Proper Motion for Blanco Cluster 1
}

\author{
Bambang Hidayat, Paulus Wiyanto \\ Bosscha Observatory and Department of Astronomy, ITB Bandung, \\ Indonesia
}

\section{Summary}

In order to establish the probability of physical membership of open clusters by means of astrometrical methods, two criteria are used: the position distribution of stars in the sky and their proper motions. Proper motion methods have shown validity from a practical point of view; but are difficult to apply where there is a high ratio of cluster-to-field stars, and in crowded fields of the Milky Way.

As a test we have selected the nearby $(170-190 \mathrm{pc})$ Blanco Cluster $1\left(1=16^{\circ}\right.$, $\mathrm{b}=-73^{\circ}$ ) in order to avoid complications arising from crowded fields. Photometric data however, suggests that the diameter of the cluster is rather extended $(\sim 120 \mathrm{arcsec})$. This will require a large-field solution, rather than a small-field solution which can be obtained with greater accuracy using Schmidt astrometry (Fresneau 1978; Nakamura \& Sekiguchi 1993).

Factors which are influential in Schmidt astrometry are:

1. Deformation of plate and emulsion in the curved plate holder.

2. Rebending to a plane during the processing and measuring.

3. Local emulsion shifts.

These may not be serious problems for our Schmidt plates because of the thinness of the plates ( 0.024 inches, due to their small size). Moreover, we developed the plates horizontally in order to avoid a downward shift of the emulsion. Magnitude dependences, which degrade positional accuracy, were eliminated by employing split-beam mode measurements on a Leitz positional measuring machine, which gave an internal accuracy of 0.1 microns. Errors in bisecting the images did occur in this mode. At the original plate scale of 162.7 $\operatorname{arcsec} / \mathrm{mm}$, the results met the accuracy described by Fresneau (1978).

The two-epoch plates, taken in 1963 and in 1979, were originally analysed on the basis of 6-parameter methods using SAO stars. 68 stars, 51 of which were identified by Epstein (1968) as cluster members, have been measured for their equatorial coordinates. The mean differences in both coordinates are:

\begin{tabular}{lcc} 
Epoch & $\mathrm{RA}\left({ }^{\prime \prime}\right)$ & $\operatorname{Dec}\left({ }^{\prime \prime}\right)$ \\
\hline 1979 & $-0.026 \pm 0.017$ & $-0.302 \pm 0.220$ \\
1963 & $-0.010 \pm 0.001$ & $-0.525 \pm 0.151$
\end{tabular}

Fig. 1 shows the resulting proper motion analysis where the majority of stars show preferential motions away from the plane. 
Following Röser (1994) the 3rd epoch plates will be taken in 1994 and 1995. These, together with other areas in the meridional fields (Palomar Groningen Fields nos. 2 and 3), will be analysed on the bases of PPM stars.

\section{Acknowledgements}

The authors are indebted to Dr. Siegfried Röser for his constructive comments. The photographic material used in this study and the Leitz measuring machine employed were generously provided by the Leids-Kerkhoven-Bosscha Foundation to the Bosscha Observatory. We record our appreciation here.

\section{References}

Epstein I., 1968, AJ, 73, 556

Fresnau A., 1978, AJ, 83, 406

Nakamura T. \& Sekiguchi M., 1993, PASJ, 45, 119

Röser S., 1994, private communication

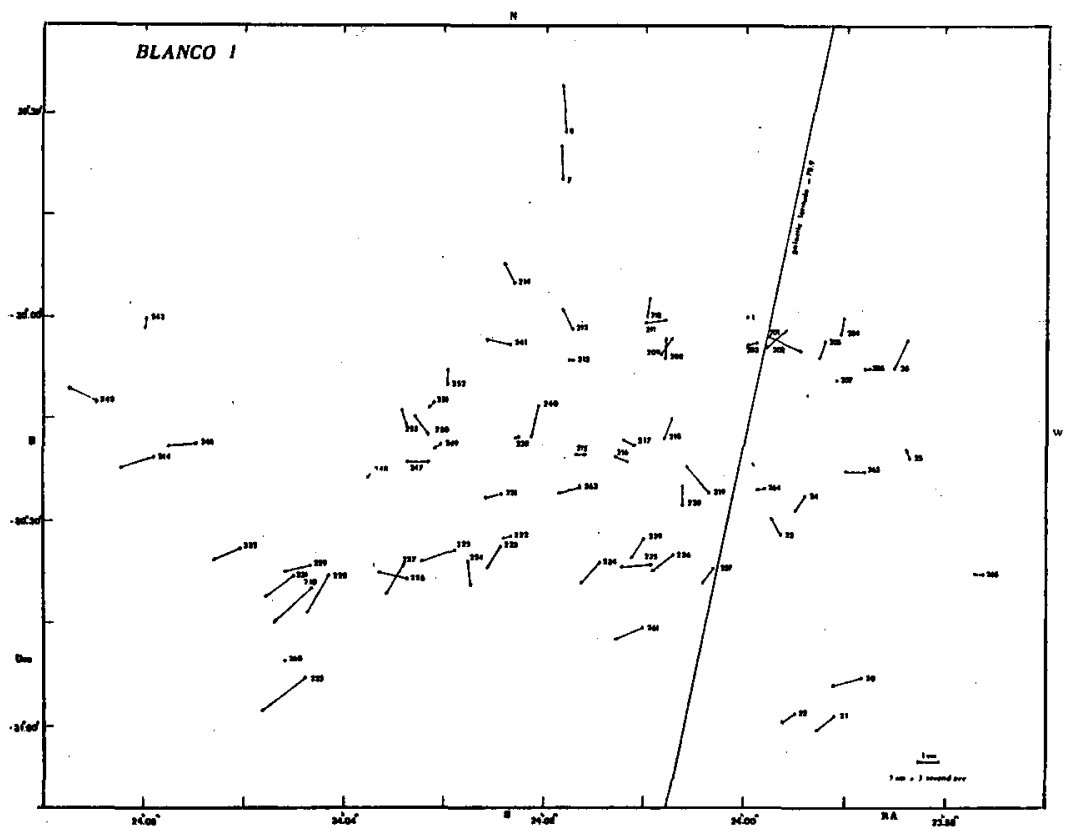

Figure 1. 